\title{
Heterosis Studies for Earliness and Yield Related Traits in Sponge Gourd [Luffa cylindrica (Roem.) L.]
}

\author{
J. Suresh Kumar ${ }^{1 *}$ and M.K. Pandit ${ }^{2}$ \\ ${ }^{1}$ ICAR-Central Tuber Crops Research Institute, Sreekariyam, \\ Thiruvananthapuram, Kerala, India \\ ${ }^{2}$ Department of Vegetable Crops, Faculty of Horticulture, BCKV, Mohanpur, \\ West Bengal, India \\ *Corresponding author
}

\section{A B S T R A C T}

\begin{tabular}{|l|}
\hline Ke y w o r d s \\
heterosis, Yield, \\
Earliness, Sex ratio \\
\hline Article Info \\
\hline $\begin{array}{l}\text { Accepted: } \\
\text { 04 August } 2019 \\
\text { Available Online: } \\
\text { 10 September } 2019\end{array}$ \\
\hline
\end{tabular}

\section{Keywords}

heterosis, Yield, Earliness, Sex ratio

Article Info

04 August 2019

10 September 2019
The present study was carried out for development of hybrids in sponge gourd for earliness and yield traits. Twenty eight crosses were developed by crossing $8 \times 8$ diallel mating design (excluding reciprocal). Evaluated the crosses and along with their parents in randomized block design (RBD). Analysis of variance indicated highly significant differences for growth, earliness and yields characters, which suggesting presence of wide genetic variability. The magnitude of standard heterosis was found in desirable direction for early and yield traits viz., days to first pistillate flower appearance in the cross IC284795 x Patna Loca (-23.07), for 50\% flowering in the cross IC-284795 x Patna Local ($18.89 \%)$, sex ratio in the cross IC-336759 x Patna Local $(-53.41 \%)$, for number of fruit per vine in the cross IC-284795 x Patna Local (34.56\%), average fruit weight in the cross IC336759 x Patna Local (110.09\%). Such crosses could be exploited for practical plant breeding programme in sponge gourd.

\section{Introduction}

Sponge gourd [Luffa cylindrica (Roem.) L.] $(2 \mathrm{n}=2 \mathrm{x}=26)$ is one of the minor cucurbitaceous vegetable crop with Old world origin in subtropical Asian region particularly India (Swarup, 2006). This crop has been cultivating for centuries in the Middle East, India, China, Japan and Malaysia (Porterfield, 1955). In India, it is cultivated on both commercial scale and in kitchen gardens during the spring-summer and rainy season (Chakravarty, 1990). The tender and immature fruits are cooked as vegetable, used in the preparation of chutneys and curries and tender fruits are easily digestible and increase appetite when consumed (Okusanya et al., 1981). Sponge gourd is a highly nutritive vegetable and contains moisture of $93.2 \mathrm{~g}$, protein $1.2 \mathrm{~g}$, fat $0.2 \mathrm{~g}$, carbohydrates $2.9 \mathrm{~g}$, vitamins (thiamine $0.02 \mathrm{mg}$, riboflavin 0.06 $\mathrm{mg}$, niacin $0.4 \mathrm{mg}$ and $\beta$ carotene $120 \mathrm{mg}$ ), minerals (calcium $36 \mathrm{mg}$, phosphorous $19 \mathrm{mg}$ and iron $1.1 \mathrm{mg}$ ) and fibers $0.20 \mathrm{~g}$ per $100 \mathrm{~g}$ of edible portion (Gopalan et al., 2012). Sponge gourd fruits contain more protein and carotene than ridge gourd (More and Shinde, 2001). 
Sponge gourd being a monoecious and cross pollinated crop, it exhibits considerable heterozygosity in population and does not suffer much due to inbreeding depression, because heterozygosity has been balanced due to primitive form of hermaphrodite flower. Crop improvement depends upon genetic architecture of yield traits and magnitude of positive heterosis towards the yield attributing traits. The wide range of genetic architecture in plant for early and yield related traits and also produce large number of hybrid seed at reasonable cost, very little work has been done to exploit the hybrid vigour in this crop. Heterosis breeding has been a recognized practical tool in providing the breeder a means of increase in yield and other economic traits in this crop. Hence, an attempt was made to study the heterosis in different crosses over better parent, best parent and the commercial check to develop and identify the suitable best performing hybrids for earliness and high yields.

\section{Materials and Methods}

Eight promising parental lines of sponge gourd namely P1 (IC-284795), P2 (IC336759), P3 (IC-284840), P4 (IC-355633), P5 (IC-544806), P6 (IC-284941), P7 (VRSG-199) and P8 (Patna local) and $28 \quad \mathrm{~F}_{1}$ hybrids obtained by crossing them in half diallel (without reciprocals), during rabi 2018 formed the experimental material. Their performance with one standard check was assessed in randomized block design (RBD) with two replications in alluvial zone of West Bengal at Horticultural Research Station, Mandouri, Bidhan Chandra Krishi Viswavidyala, West Bengal, India. Distance between rows was kept $100 \mathrm{~cm}$ and plants were spaced at $50 \mathrm{~cm}$ apart within the row. Observations were recorded on five randomly selected plants from each replication on vine length, number of primary branches, number of fruiting nodes on main stem, days to first staminate flower appearance, days to first pistillate flower appearance, days to $50 \%$ flowering, span of flowering, sex ratio, node of 1 st female flower appearance, days to harvestable maturity from anthesis, fruit length, fruit diameter, fruit weight, fruits per plant, number of seeds per fruit, seed index (100 seed weight), yield per plant. Heterosis was calculated as percentage of $F_{1}$ over better parent and standard check Pusa chikni. The formula used to estimate heterosis over better parent and standard check was as per Fonseca and Patterson (1968).

\section{Results and Discussion}

The analysis of variance for experimental design (Table 1) revealed highly significant mean squares differences due to genotypes, parents and hybrids for all the characters indicating sufficient amount of genetic variability of parents and hybrids for the 17 traits studied. The aim of heterosis study is to identifying the best heterotic combinations and its exploitation for commercial purpose. $\mathrm{Per}$ se performance and range (mean performance) of parents and crosses for various heterotic effects as well as heterotic responses of $F_{1}$ hybrids over mid parent (MP), better parent (BP) and standard check (CC) for 17 characters; and the best heterotic crosses identified on the basis of earliness and yield parameters are presented in the table 2 and 3.

In the present investigation, fruit yield per vine was found to be the most heterotic trait as heterosis for fruit yield per vine ranged from 42.93 to $66.01 \%,-62.71$ to $51.99 \%$ and -76.02 to $127.74 \%$ over mid parent, better parent and standard check respectively (Table 2). The magnitude of heterotic effects were high for fruit yield per vine, fruit length, fruit diameter, individual fruit weight, seed index and number of fruiting nodes on main stem, while significant heterosis was low to moderate for rest of the traits. The cross IC-336759 x Patna local showed significant and positive standard 
heterosis $(127.74 \%)$ for fruit yield per vine followed by cross IC-284795 x Patna local $(120.20 \%)$ and IC-284795 $x$ IC-336759 $(94.83 \%)$ (Table 3$)$. In such crosses expression of heterotic response over better parent and standard check indicates the real superiority of hybrids from the commercial point of view and these hybrids can be commercially exploited after large scale testing. These results are akin to the findings of Muthaiah et al., (2017), Bairwa et al., (2017), Poshiya et al., (2015), Prakash et al., (2014), Narasannavar et al., (2014), Karthik (2014), Lodam et al., (2014), Dodiya et al., (2013) and Singh (2013) have reported high values of heterosis for fruit yield in ridge gourd. Sonavane et al., (2013), Islam et al., (2012), Sanandia et al., (2008) and Naliyadhara et al., (2007) in sponge gourd.

Other yield related parameters like fruit length, fruit diameter, average fruit weight and number of fruits per plant, etc. are desirable features in sponge gourd cultivation. Therefore, significant and positive heterosis for these characters is desirable. The cross IC336759 x Patna local showed significant and positive standard heterosis $(44.07 \%)$ for fruit length followed by cross IC-284840 x IC$355633(42.75 \%)$ and IC-336759 x VRSG-199 (39.90\%). The cross IC-284795 x Patna local showed significant and positive standard heterosis (148.72\%) for fruit diameter followed by cross IC-284840 x Patna local $(136.29 \%)$ and IC-336759 x Patna local (129.59\%). The cross IC-336759 x Patna local showed significant and positive standard heterosis (110.09\%) for average fruit weight followed by cross IC-284795 x Patna local $(93.50 \%)$ and IC-336759 xVRSG-199 (88.72\%). The cross IC-284795 x Patna local showed significant and positive standard heterosis $(34.56 \%)$ for number of fruits per plant followed by cross IC-284795 x IC336759 (31.00\%) and IC-336759 x Patna local $(27.04 \%)$.
Early flowering, lower nodal position of flowers, early picking and lower sex ratio are desirable features in sponge gourd cultivation. Therefore, significant and negative heterosis for these characters is desirable. For days to first female flower appearance maximum and significant negative heterosis over better parent was observed in the cross IC-284941 x VRSG-199 (-32.08\%) followed by IC-355633 $x$ Patna local (-29.89\%) and IC-284941 x Patna local (-28.08\%); negative heterosis over standard check was observed in the cross IC284795 x Patna local $(-18.89 \%)$ followed by IC-284795 x IC-336759 (-16.42\%) and IC284941 x VRSG-199 (-12.66\%). For lower sex ratio maximum and significant negative heterosis over better parent was observed in the cross IC- 336759 x Patna local (-52.07) followed by VRSG-199 x Patna local (-49.78) and IC-284840 x Patna local (-44.55); negative heterosis over commercial check was observed in the cross IC-336759 x Patna local (-53.41) followed by IC-284795 x Patna local $(-40.09)$ and IC-284840 x Patna local (-35.85). For node at first female flower appearance maximum and significant negative heterosis over better parent was observed in the cross IC-355633 x Patna local (-52.19) followed by IC-544806 Patna local (-47.24) and IC-284795 $\mathrm{x}$ Patna local (-35.83); negative heterosis over commercial check was observed in the cross IC-336759 x Patna local (-46.82) followed by IC-284795 x Patna local (-45.45) and IC$284795 \times$ IC-336759 (-40.91). For the character on days to harvestable maturity significant negative heterosis over better parent was observed in the cross VRSG-199 $\mathrm{x}$ Patna local (-40.29) followed by IC-355633 x Patna local (-40.00) and IC-284840 x Patna local (-35.56); negative heterosis over commercial check was observed in the cross IC-336759 x Patna local (-12.30) followed by VRSG-199 x Patna local (-8.47) and IC284795 x IC-336759 (-7.70). 
Table.1 Analysis of variance for seventeen characters in $8 \times 8$ diallel cross (without reciprocals) in sponge gourd

\begin{tabular}{|c|c|c|c|c|c|c|c|}
\hline \multirow[t]{2}{*}{ Sl.NO } & \multirow[t]{2}{*}{ Character } & \multicolumn{6}{|c|}{ Mean sum of square } \\
\hline & & Replication & Genotypes & Parents & Hybrids & Parents Vs Hybrids & Error \\
\hline & df & 2 & 35 & 7 & 27 & 1 & 70 \\
\hline 1 & Vine length (m)(90 DAS) & 0.0013 & $3.5747 * *$ & $2.4244 * *$ & $3.2857 * *$ & $19.4276 * *$ & 0.0215 \\
\hline 2 & No. of primary branches & 0.0109 & $5.2561 * *$ & $3.3691 * *$ & $4.8961 * *$ & $28.1862 * *$ & 0.0500 \\
\hline 3 & No. of fruiting nodes on main stem & 0.0762 & $9.8931 * *$ & $10.9128 * *$ & $8.2420 * *$ & $47.3362 * *$ & 0.0792 \\
\hline 4 & Days to first staminate flower appearance & 2.4901 & $77.8996 * *$ & $70.8257 * *$ & $66.4901 * *$ & $435.4728 * *$ & 2.1578 \\
\hline 5 & Days to first pistillate flower appearance & 0.1330 & $113.9509 * *$ & $105.7898 * *$ & $103.0003 * *$ & $466.7444 * *$ & 0.6700 \\
\hline 6 & Days to $50 \%$ flowering & 0.4811 & $126.2748 * *$ & $112.0302 * *$ & $93.2411 * *$ & $1117.8969 * *$ & 0.7283 \\
\hline 7 & Span of flowering & 0.1193 & $162.1880 * *$ & $104.6576 * *$ & $153.6242 * *$ & $796.1231 * *$ & 0.6427 \\
\hline 8 & Sex ratio $(\mathbf{M} / \mathbf{F})$ & 0.4294 & $64.8522 * *$ & $45.8872 * *$ & $60.9544 * *$ & $302.8501 * *$ & 0.2652 \\
\hline 9 & Node of $1^{\text {st }}$ female flower appearance & 0.0138 & $27.0008 * *$ & $38.1359 * *$ & $23.8305 * *$ & $34.6529 * *$ & 0.0668 \\
\hline 10 & Days to harvestable maturity from anthesis & 0.0424 & $11.5035^{* *}$ & $4.6537 * *$ & $12.0885^{* *}$ & $43.6560 * *$ & 0.0186 \\
\hline 11 & Fruit length (cm) & 0.0407 & $109.5278^{* *}$ & $98.6901 * *$ & $92.9672 * *$ & $632.5306^{* *}$ & 0.1415 \\
\hline 12 & Fruit diameter $(\mathrm{cm})$ & 0.2497 & $15.6355^{* *}$ & $6.2459 * *$ & $17.1107 * *$ & $41.5313 * *$ & 0.1228 \\
\hline 13 & Average fruit weight (g) & 3.0589 & $6277.6372 * *$ & $4829.4780 * *$ & $5933.8525 * *$ & $25696.9434 * *$ & 5.0996 \\
\hline 14 & Fruits per plant & 0.1499 & $35.6331 * *$ & $30.7599 * *$ & $35.5249 * *$ & $72.6669 * *$ & 0.1283 \\
\hline 15 & No. of seeds per fruit & 0.9628 & $2459.9255^{* *}$ & $2088.7559 * *$ & $2554.4856 * *$ & $2504.9861 * *$ & 1.4259 \\
\hline 16 & Seed index (100 seed weight) & 0.0020 & $11.4730 * *$ & $9.5163 * *$ & $11.3336^{* *}$ & $28.9366^{* *}$ & 0.1016 \\
\hline 17 & Yield per plant (kg) & 0.0010 & $3.9514 * *$ & $2.5734 * *$ & $3.9918 * *$ & $12.5060 * *$ & 0.0060 \\
\hline
\end{tabular}

$*$ : Significant at $\mathrm{p}=0.05, * *$ Significant at $\mathrm{p}=0.01$ 
Table.2 Range and heterosis for growth and yield characters in sponge gourd

\begin{tabular}{|c|c|c|c|c|c|c|}
\hline \multirow[t]{2}{*}{ Sl.NO } & \multirow[t]{2}{*}{ Character } & \multicolumn{2}{|c|}{ Per se performance (range) } & \multicolumn{3}{|c|}{ Heterosis (\%) } \\
\hline & & Parents & Crosses & MP & BP & $\mathbf{C C}$ \\
\hline 1 & Vine length (m)(90 DAS) & 2.25 to 4.80 & 2.68 to 6.23 & 9.92 to 55.09 & -14.06 to 32.98 & -27.22 to 69.02 \\
\hline 2 & No. of primary branches & 2.50 to 5.50 & 1.55 to 7.00 & -46.09 to 63.35 & -52.31 to 42.97 & -61.73 to 72.84 \\
\hline 3 & $\begin{array}{l}\text { No. of fruiting nodes on main } \\
\text { stem }\end{array}$ & 2.85 to 7.20 & 2.00 to 8.95 & -31.58 to 73.42 & -33.33 to 40.14 & -68.99 to 38.76 \\
\hline 4 & $\begin{array}{l}\text { Days to first staminate flower } \\
\text { appearance }\end{array}$ & 34.00 to 48.00 & 28.35 to 45.35 & -23.91 to 8.11 & -33.37 to 2.86 & -26.36 to 17.80 \\
\hline 5 & $\begin{array}{l}\text { Days to first pistillate flower } \\
\text { appearance }\end{array}$ & 40.00 to 56.35 & 33.35 to 55.00 & -22.32 to 8.25 & -31.06 to 4.39 & -23.07 to 26.87 \\
\hline 6 & Days to $50 \%$ flowering & 49.50 to 66.25 & 41.00 to 63.36 & -27.02 to -2.65 & -32.08 to -5.58 & -18.89 to 25.34 \\
\hline 7 & Span of flowering & 32.65 to 52.00 & 31.14 to 60.00 & -11.22 to 29.32 & -16.96 to 24.38 & -15.95 to 61.94 \\
\hline 8 & Sex ratio $(M / F)$ & 14.5 to 27.00 & 8.32 to 29.00 & -47.48 to 14.85 & -52.07 to 7.41 & -53.41 to 62.46 \\
\hline 9 & $\begin{array}{l}\text { Node of } 1^{\text {st }} \text { female flower } \\
\text { appearance }\end{array}$ & 6.50 to 16.00 & 5.85 to 17.00 & -32.00 to 10.49 & -52.19 to -9.87 & -46.82 to 54.55 \\
\hline 10 & $\begin{array}{l}\text { Days to harvestable maturity } \\
\text { from anthesis }\end{array}$ & 7.00 to 10.65 & 5.35 to 14.55 & -31.70 to 37.59 & -40.29 to 36.62 & -12.30 to 138.52 \\
\hline 11 & Fruit length $(\mathrm{cm})$ & 11.50 to 27.00 & 13.10 to 32.80 & -8.43 to 94.90 & -6.04 to 48.74 & -42.46 to 44.07 \\
\hline 12 & Fruit diameter $(\mathrm{cm})$ & 4.75 to 9.00 & 4.05 to 13.00 & -36.06 to 73.58 & -40.00 to 44.44 & -22.51 to 148.72 \\
\hline 13 & Average fruit weight (g) & 115.50 to 235 & 102.25 to 298.85 & -16.82 to 40.94 & -44.55 to 29.57 & -28.12 to 110.09 \\
\hline 14 & Fruits per plant & 5.36 to 13.58 & 4.55 to 17.00 & -26.81 to 56.78 & -37.54 to 25.86 & -63.98 to 34.56 \\
\hline 15 & No. of seeds per fruit & 60 to 130 & 54.00 to 165.15 & -22.67 to 47.47 & -36.44 to 27.04 & -48.82 to 56.54 \\
\hline 16 & Seed index (100 seed weight) & 9.65 to 14.35 & 7.55 to 15.95 & -22.56 to 24.34 & -23.35 to 20.78 & -38.37 to 30.20 \\
\hline 17 & Yield per plant (kg) & 0.62 to 3.19 & 0.51 to 4.84 & -42.93 to 66.01 & -62.71 to 51.99 & -76.02 to 127.74 \\
\hline
\end{tabular}

$\mathrm{MP}=$ mid parent, $\mathrm{BP}=$ better parent and $\mathrm{CC}=$ standard check 
Table.3 Promising hybrids with favourable heterosis for seventeen characters

\begin{tabular}{|c|c|c|c|c|}
\hline \multirow{2}{*}{$\begin{array}{l}\text { S. } \\
\text { No }\end{array}$} & \multirow[t]{2}{*}{ Chara-cter } & \multicolumn{3}{|c|}{ Top three crosses with significant Heterosis } \\
\hline & & Heterosis (\%) & Heterobeltiosis (\%) & Standard Heterosis (\%) \\
\hline 1 & VL & $\begin{array}{l}\text { IC-544806 xVRSG-199 (55.09) } \\
\text { IC-336759 x IC-355633 (47.91) } \\
\text { IC-336759 xVRSG-199 (44.93) }\end{array}$ & $\begin{array}{l}\text { IC-544806 xVRSG-199 }(32.98) \\
\text { IC-336759 xVRSG-199 }(31.40) \\
\text { IC-336759 x PATNA LOCAL }(29.86)\end{array}$ & $\begin{array}{l}\text { IC-336759 } \times \text { PATNA LOCAL }(69.08) \\
\text { IC- } 284795 \times \text { PATNA LOCAL }(60.94) \\
\text { IC- } 284840 \times \text { PATNA LOCAL }(55.88)\end{array}$ \\
\hline 2 & PB & $\begin{array}{l}\text { IC- } 284840 \times \text { IC-355633 }(65.35) \\
\text { IC-284795 x PATNA LOCAL }(35.31) \\
\text { IC- } 284840 \times \text { PATNA LOCAL }(29.24)\end{array}$ & $\begin{array}{l}\text { IC-284840 x IC-355633 (30.41) } \\
\text { IC-284795 x VRSG-199 (37.04) } \\
\text { IC-284795 x IC-336759 (34.93) }\end{array}$ & $\begin{array}{l}\text { IC-284795 x PATNA LOCAL }(72.84) \\
\text { VRSG-199 x PATNA LOCAL }(67.98) \\
\text { IC-284795 x IC-336759 }(66.58)\end{array}$ \\
\hline 3 & FN & $\begin{array}{l}\text { IC-544806 xVRSG-199 (73.42) } \\
\text { IC-355633 xVRSG-199 (59.30) } \\
\text { IC-355633 x PATNA LOCAL (59.26) }\end{array}$ & $\begin{array}{l}\text { IC-544806 x IC-284941 (40.14) } \\
\text { IC-544806 xVRSG-199 (38.73) } \\
\text { IC-284840 x VRSG-199 (25.65) }\end{array}$ & $\begin{array}{l}\text { IC-336759 x PATNA LOCAL }(38.76) \\
\text { IC-284795 x IC-336759 }(35.71) \\
\text { IC- } 284795 \times \text { xATNA LOCAL }(31.78)\end{array}$ \\
\hline 4 & DSF & $\begin{array}{l}\text { IC-284941x PATNA LOCAL (-23.91) } \\
\text { IC-284941x VRSG-199 (-23.52) } \\
\text { IC-544806 x PATNA LOCAL }(-21.93)\end{array}$ & $\begin{array}{l}\text { IC-284941x PATNA LOCAL }(-33.37) \\
\text { IC-355633 x PATNA LOCAL }(-32.81) \\
\text { IC-544806 x PATNA LOCAL }(-30.62)\end{array}$ & $\begin{array}{l}\text { IC-336759 x PATNA LOCAL }(-26.36) \\
\text { IC-284795 x PATNA LOCAL }(-23.38) \\
\text { IC-284941x PATNA LOCAL }(-21.69)\end{array}$ \\
\hline 5 & DFF & $\begin{array}{l}\text { IC-284941x VRSG-199 (-22.32) } \\
\text { IC-284795 x IC-355633 (-21.24) } \\
\text { IC-544806 x IC-284941 (-20.10) }\end{array}$ & $\begin{array}{l}\text { IC-284795 x IC-355633 (-31.06) } \\
\text { IC-355633 x PATNA LOCAL (-30.79) } \\
\text { IC-284941x PATNA LOCAL }(-28.80)\end{array}$ & $\begin{array}{l}\text { IC-284795 x PATNA LOCAL }(-23.07) \\
\text { IC-336759 x PATNA LOCAL (-21.91) } \\
\text { IC-284795 x IC-336759 (-18.02) }\end{array}$ \\
\hline 6 & DPF & $\begin{array}{l}\text { IC-284941x VRSG-199 (-27.02) } \\
\text { IC-355633 x PATNA LOCAL (-19.74) } \\
\text { IC-544806 x IC-284941 (-19.59) }\end{array}$ & $\begin{array}{l}\text { IC-284941x VRSG-199 (-32.08) } \\
\text { IC-355633 x PATNA LOCAL (-29.89) } \\
\text { IC-284941x PATNA LOCAL }(-28.08)\end{array}$ & $\begin{array}{l}\text { IC-284795 x PATNA LOCAL (-18.89) } \\
\text { IC-284795 x IC-336759 (-16.42) } \\
\text { IC-284941x VRSG-199 (-12.66) }\end{array}$ \\
\hline 7 & SF & $\begin{array}{l}\text { IC-336759 x IC-355633 }(29.32) \\
\text { IC-355633 x PATNA LOCAL }(26.74) \\
\text { IC-284840 x IC-544806 }(25.95)\end{array}$ & $\begin{array}{l}\text { IC-284840 x IC-544806 (24.38) } \\
\text { IC-284795 x IC-336759 (21.14) } \\
\text { IC-336759 x VRSG-199 (16.82) }\end{array}$ & $\begin{array}{l}\text { IC-336759 x PATNA LOCAL (61.4) } \\
\text { IC-284795 x PATNA LOCAL }(57.89) \\
\text { IC- } 284840 \text { x PATNA LOCAL }(55.81)\end{array}$ \\
\hline 8 & SR & $\begin{array}{l}\text { IC-336759 x PATNA LOCAL }(-47.78) \\
\text { VRSG-199 x PATNA LOCAL }(-38.40) \\
\text { IC-284795 x PATNA LOCAL }(-36.39)\end{array}$ & $\begin{array}{l}\text { IC-336759 x PATNA LOCAL }(-52.07) \\
\text { VRSG-199 x PATNA LOCAL }(-49.78) \\
\text { IC-284840 x PATNA LOCAL }(-44.55)\end{array}$ & $\begin{array}{l}\text { IC-336759 x PATNA LOCAL }(-53.41) \\
\text { IC-284795 x PATNA LOCAL }(-40.09) \\
\text { IC-284840 x PATNA LOCAL }(-35.85)\end{array}$ \\
\hline 9 & NFA & $\begin{array}{l}\text { IC-355633 x PATNA LOCAL }(-32.00) \\
\text { IC-284795 x IC-284840 (-27.65) } \\
\text { IC-284795 x IC-336759 (-25.07) }\end{array}$ & $\begin{array}{l}\text { IC-355633 x PATNA LOCAL }(-52.19) \\
\text { IC-544806 x PATNA LOCAL }(-47.24) \\
\text { IC-284795 x PATNA LOCAL }(-35.83)\end{array}$ & $\begin{array}{l}\text { IC-336759 x PATNA LOCAL }(-46.82) \\
\text { IC-284795 x PATNA LOCAL }(-45.45) \\
\text { IC-284795 x IC-336759 }(-40.91)\end{array}$ \\
\hline 10 & DHM & $\begin{array}{l}\text { VRSG-199 x PATNA LOCAL }(-31.70) \\
\text { IC-284795 x IC-336759 (-31.31) } \\
\text { IC-284840 x IC-544806 (-30.08) }\end{array}$ & $\begin{array}{l}\text { VRSG-199 x PATNA LOCAL }(-40.29) \\
\text { IC-355633 x PATNA LOCAL }(-40.00) \\
\text { IC-284840 x PATNA LOCAL }(-35.56)\end{array}$ & $\begin{array}{l}\text { IC-336759 x PATNA LOCAL }(-12.30) \\
\text { VRSG-199 x PATNA LOCAL }(-8.47) \\
\text { IC- } 284795 \times \text { IC-336759 }(-7.70)\end{array}$ \\
\hline 11 & FL & IC-284840 x IC-355633 (94.90) & IC-284840 x IC-355633 (48.74) & IC-336759 x PATNA LOCAL (44.07) \\
\hline
\end{tabular}




\begin{tabular}{|c|c|c|c|c|}
\hline & & $\begin{array}{l}\text { IC-355633 xVRSG-199 }(57.95) \\
\text { IC-355633 x PATNA LOCAL }(53.25)\end{array}$ & $\begin{array}{l}\text { IC-284840 x VRSG-199 (31.53) } \\
\text { IC-336759 x VRSG-199 (25.23) }\end{array}$ & $\begin{array}{l}\text { IC-284840 x IC-355633 (42.75) } \\
\text { IC-336759 xVRSG-199 (39.90) }\end{array}$ \\
\hline 12 & FD & $\begin{array}{l}\text { IC-284840 x PATNA LOCAL (73.58) } \\
\text { IC-284795 x PATNA LOCAL (65.08) } \\
\text { IC-284795 x VRSG-199 (55.65) }\end{array}$ & $\begin{array}{l}\text { IC-284795 x PATNA LOCAL }(44.44) \\
\text { IC-284840 x PATNA LOCAL }(37.22) \\
\text { IC-544806 x IC-284941 (35.72) }\end{array}$ & $\begin{array}{l}\text { IC-284795 x PATNA LOCAL (148.72) } \\
\text { IC-284840 x PATNA LOCAL (136.29) } \\
\text { IC-336759 x PATNA LOCAL (129.59) }\end{array}$ \\
\hline 13 & FW & $\begin{array}{l}\text { IC-355633 x PATNA LOCAL (40.94) } \\
\text { IC-355633 xVRSG-199 (40.57) } \\
\text { IC-336759 xVRSG-199 (39.33) }\end{array}$ & $\begin{array}{l}\text { IC-284840 x VRSG-199 (29.57) } \\
\text { IC-336759 x PATNA LOCAL (27.17) } \\
\text { IC-336759 xVRSG-199 (26.63) }\end{array}$ & $\begin{array}{l}\text { IC-336759 x PATNA LOCAL (110.09) } \\
\text { IC-284795 x PATNA LOCAL (93.50) } \\
\text { IC-336759 xVRSG-199 (88.72) }\end{array}$ \\
\hline 14 & FPP & $\begin{array}{l}\text { IC-355633 x PATNA LOCAL }(56.78) \\
\text { IC-284941x PATNA LOCAL }(36.58) \\
\text { IC-544806 x PATNA LOCAL }(35.55)\end{array}$ & $\begin{array}{l}\text { IC-284795 x IC-336759 }(25.86) \\
\text { IC-284795 x PATNA LOCAL }(25.15) \\
\text { IC-284795 x IC-284840 }(19.62)\end{array}$ & $\begin{array}{l}\text { IC-284795 x PATNA LOCAL }(34.56) \\
\text { IC-284795 x IC-336759 }(31.00) \\
\text { IC-336759 x PATNA LOCAL }(27.04)\end{array}$ \\
\hline 15 & NSF & $\begin{array}{l}\text { IC-355633 xVRSG-199 }(47.47) \\
\text { IC-355633 x PATNA LOCAL }(46.68) \\
\text { IC-284941x VRSG-199 }(35.38)\end{array}$ & $\begin{array}{l}\text { IC-284795 x PATNA LOCAL }(27.04) \\
\text { IC-544806 x IC-284941 }(24.98) \\
\text { VRSG-199 x PATNA LOCAL }(18.85)\end{array}$ & $\begin{array}{l}\text { IC- } 284795 \times \text { PATNA LOCAL }(56.54) \\
\text { VRSG-199 x PATNA LOCAL }(46.45) \\
\text { IC-336759 } \times \text { PATNA LOCAL }(41.23)\end{array}$ \\
\hline 16 & SI & $\begin{array}{l}\text { IC-355633 x PATNA LOCAL }(24.34) \\
\text { IC-355633 x VRSG-199 (23.47) } \\
\text { IC-336795 x IC-336759 (21.29) }\end{array}$ & $\begin{array}{l}\text { IC-355633 x VRSG-199 (20.78) } \\
\text { IC-284795 x IC-336759 (19.92) } \\
\text { IC-544806 x IC-284941 (10.50) }\end{array}$ & $\begin{array}{l}\text { IC- } 284795 \text { x IC-336759 }(30.20) \\
\text { IC-355633 x PATNA LOCAL }(28.65) \\
\text { IC- } 284795 \text { x PATNA LOCAL }(27.76)\end{array}$ \\
\hline 17 & $\mathrm{Y}$ & $\begin{array}{l}\text { IC-355633 x PATNA LOCAL }(92.82) \\
\text { IC-336759 x PATNA LOCAL }(66.01) \\
\text { IC-284941 x VRSG-199 }(65.03)\end{array}$ & $\begin{array}{l}\text { IC-336759 x PATNA LOCAL }(51.98) \\
\text { IC-284795 x IC-336759 }(48.51) \\
\text { IC-284795 x PATNA LOCAL }(46.97)\end{array}$ & $\begin{array}{l}\text { IC-336759 } \times \text { PATNA LOCAL }(127.74) \\
\text { IC- } 284795 \times \text { PATNA LOCAL }(120.2) \\
\text { IC- } 284795 \times \text { IC-336759 }(94.83)\end{array}$ \\
\hline
\end{tabular}

(VL)- Vine length (m); (PB)-No. of primary branches; (FN)-Number of fruiting nodes on main stem; (DSF)-Days to first staminate flower appearance; (DPF)Days to first pistillate (female) flower appearance; (DFF)-Days to 50\% flowering; (SF)-Span of flowering; (SR)-Sex ratio (M/F); (NFA)-Node of 1st female flower appearance; (DHM)-Days to harvestable maturity from anthesis; (FL)-Fruit length (cm); (FD)-Fruit diameter (cm); (FW)-Average fruit weight (g); (FPP)Fruits per plant (no); (NSF)- Number of seeds per fruit; (SI)-Seed index (100 seed weight); (Y)- Yield per plant (kg). 
Similar results were reported for the earliness of above mentioned characters by Sonavane et al., (2013), Islam et al., (2012), Sanandia et al., (2008) and Naliyadhara et al., (2007) in sponge gourd; and Muthaiah et al., (2017), Bairwa et al., (2017), Prakash et al., (2014), Narasannavar et al., (2014), Karthik (2014), Lodam et al., (2014), Dodiya et al., (2013) and Singh (2013) in ridge gourd.

In conclusion the results discussed above are quite indicative of the fact that hybrids in sponge gourd have greater potential for maximizing yields with earliness. Based on heterosis study the $\mathrm{F}_{1}$ hybrids IC-284795 $\mathrm{x}$ Patna Local, IC-336759 x Patna Local and IC-284795 x IC-336759 were found best performers keeping in view the earliness as well as increased yield over standard check and their respective better parent and may be entered in multi-locational trials.

\section{References}

Bairwa, S. K., Soni, A. K., and Shekhawat, A. S., 2017. Heterosis studies in ridge gourd [Luffa acutangula (Roxb.) L.]. International Journal of Current Microbiology and Applied Sciences, 6(11): 1572-1576.

Chakravarty, H. L., 1990. Cucurbits of India and their role in the development of vegetable crops. In: Biology and utilization of Cucurbitaceae. (Eds.) Bates, D. M., Robinson, R. W. and Jeffery, C. Cornell University Press, Ithaca, New York (U.S.A).

Chauhan, D. V. S., 1972. Vegetable production in India. Ram Prasad and Sons, Agra.

Dodiya, A. J., Bhatiya, V. J., Kulkarni, G. U., Sharma, L. K., and Sanjeev Kumar., 2013. Heterosis and combining ability for fruit yield and yield components in ridge gourd (Luffa acutangula (Roxb.) L.). Progressive Research, 8(2):179-184
Fonseca, S., and Patterson, F. L., 1968. Hybrid vigour in seven parent diallel cross in common winter wheat (Triticum aestivam L.). Crop Sci., 8: 8595.

Gopalan, C., Sastri, V., Balasubramanium, S. C., Rao, B. S. N., Dosthale, Y. G., and Pant, K. C., 2012. Nutritive value of Indian foods. Indian Council of Medical Research Technological Bulletin. National Institute of Nutrition (NIN), Hyderabad. p 51.

Islam, S., Munshi, A. D., and Kumar, R., 2012. Studies on heterosis and combining ability for earliness and yield in sponge gourd. Indian Journal of Horticulture, 69(3): 348-352.

Karthik R. P., 2014. Heterosis studies in ridge gourd (Luffa acutangula L.). 2nd International Conference on Agricultural and Horticultural Sciences. Hyderabad, 03-05 Feb, 2014.

Lodam, V. A., Patil, P. P., and Desai, D. T., 2014. Exploitation of hybrid vigour in ridge gourd (Lufa acutangula Roxb.). Electronic Journal of Plant Breeding, 5(4): 792-796.

More, T.A, and Shinde, K. G., 2001. Ridge and Sponge gourd. In: Vegetables, tuber crops and spices. (Eds.) Thamburaj, S. and Singh, N. Indian Council of Agricultural Research. p 309.

Muthaiah, K., Gasti, V. D., Mallesh Sanganamoni., and Nagaraju Kattula., 2017. Heterosis studies for earliness and yield related traits in ridge gourd [Luffa acutangula (L.) Roxb.]. International Journal of Current Microbiology and Applied Sciences, 6(6): 2656-2661.

Naliyadhara, M. V., Dhaduk, L. K., Barad, A. V., Purohit, V. L and Vachhani, J. H. 2007. Heterosis for fruit yield and its components in sponge gourd [Luffa cylindrical (Roem.) L.]. National Journal of Plant Improvement, 9(2):132-135. 
Narasannavar, A. R., Gasti, V. D., Shantappa, T., Mulge, R., Allolli, T. B., and Thammaiah, N. 2014. Heterosis studies in ridge gourd [Luffa acutangula (L.) Roxb.]. Karnataka Journal of Agriculture Sciences, 27 (1): 47-51.

Okusanya, O. T, Ola., Adams, B. A., and Bamidele, J. F., 1981. Variation in size, leaf morphology and fruit characters among twenty five populations of Luffa aegyptica. Canadian Journal of Botany, 59: 2618-2627.

Porterfield, W. M., 1955. Loofah: the sponge gourd. Econ. Bot., 9: 211-223.

Poshiya, S. C., Dhaduk, L. K., Lata Raval., and Mehta, D. R., 2015. Heterosis study in ridge gourd (Luffa acutangula (Roxb.) L.). Electronic Journal of Plant Breeding, 6(2): 581-585.

Prakash, M., Jagadeesha, R. C., Savitha, H. N., and Kumara, B. R., 2014. Performance of parents and hybrids for yield attributing characters in ridge gourd (Luffa acutangula (Roxb.) L.). Trends in Biosciences, 7(22): 36063610.

Sanandia, S. T., Mehta, D. R, Tarpara, V. D., and Dhaduk, L. K., 2008. Studies on relative heterosis, heterobeltiosis and inbreeding depression on fruit yield and its attributes in sponge gourd [Luffa cylindrica (L.)Roem.]. National Journal of Plant Improvement, 1:8-12.

Singh, J., 2013. Combining ability, gene action and heterosis studies in ridge gourd (Luffa acutangula L.) Roxb. M.Sc thesis, IARI, New Delhi, India.

Sonavane, P. N, Bhalekar, M. N., Pawar, P. K., and Bawkar, S. O., 2013. Studies on heterosis and combining ability for earliness and yield in sponge gourd. Bioinfolet, 10(2): 662-666.

Swarup,V., 2006. Cucurbits, In: Vegetable Science and Technology in India. Kalyani Publishers, Kolkata. p 426.

\section{How to cite this article:}

Suresh Kumar, J. and Pandit, M.K. 2019. Heterosis Studies for Earliness and Yield Related Traits in Sponge Gourd [Luffa cylindrica (Roem.) L.]. Int.J.Curr.Microbiol.App.Sci. 8(09): 169-177. doi: https://doi.org/10.20546/ijcmas.2019.809.022 